\title{
Dupuytren Contracture in a Patient with Congenital Camptodactyly and Incidental Carpal Tunnel Syndrome
}

\author{
S Mahendran, MBBS, H Paisal, MBBS, ES Ng, MS (Ortho), T Sara, FRCS \\ Department of Orthopaedics, University Malaya Medical Centre, Kuala Lumpur, Malaysia
}

\begin{abstract}
Dupuytren contracture is commonly seen in northern European populations but not in Asians. Even more rare is a presentation of flexion deformity of fingers involving two different pathologies with incidental carpal tunnel syndrome in the same patient. We report herein a case of Dupuytren contracture with congenital camptodactyly and unilateral carpal tunnel syndrome.
\end{abstract}

Key Words:

Dupuytren's contracture, Camptodactyly, Carpal tunnel syndrome

\section{CASE REPORT}

A 52-year-old Punjabi gentleman, presented with history of progressive flexion deformity on his right middle finger over the previous four months. The deformity progressively worsened and interfered with his daily activities by the time of his first visit. Although he first noticed the deformity when resting his palm on a table, the condition progressed to the point that even shaking hands had become difficult. The patient also reported progressively worsening numbness that started with the middle finger and extended over the lateral four digits. History was remarkable for a fracture over the fifth metacarpal at about ten years of age that was treated conservatively with a splint. This patient also had flexion deformities of both little fingers that were present since birth for which no treatment was sought. He consumed alcohol two bottles of beer two to three times a week and smoked 20 -30 cigarettes a day for the past 20 years. There was no history of diabetes or other medical conditions. The patient has a brother with Dupuytren contracture of the right middle finger who was doing well after surgical correction.

Upon examination, the patient had bilateral camptodactyly with flexion at the metacarpal phalangeal (MCP) and proximal interphalangeal (PIP) joints of the little fingers. There were no cords palpable over the palm in line with these fingers. The PIP joint was flexed at 100 degrees and MCP joint flexed at 30 degrees. There was a also flexion deformity of the right middle finger at the MCP joint of 45 degrees and PIP joint of 70 degrees (figure 1). Thick cords were palpable over the palm along the middle finger with blanching of skin on extension and puckering over the lateral aspect of the middle finger at the PIP joint (figure 2). Range of motion was full at the DIP joint, 70 - 100 degrees at PIP joint and 45 - 90 degrees at the MCP joint. Tinel's sign and Phalen's test were also positive. There was no thenar muscle wasting seen, but sensory examination revealed reduced sensation over the thumb, index, and middle and radial half of the ring fingers but more marked reduction over the middle finger especially over the radial border. Sensation over the thenar eminence was intact. No knuckle pads, plantar nodules or deformities of the penis were seen.

Regional fasciectomy was performed using a single zigzag palmar incision incorporated with a carpal tunnel release incision. Intra operatively, the flexor retinaculum was noted to be thickened. The pre-tendinous cord (figure 3) extended distal to transverse over the metacarpal ligament as a spiral cord curving around the lateral margin of the proximal phalanx to the level of the PIP joint (figure 4). The radial digital artery and nerve were displaced to the midline by the spiral cord (figure 4). These structures were carefully dissected and released. Tourniquet was subsequently released and careful haemostasis secured before skin closure. Postoperatively the patient's hand was splinted with the fingers in extension and the wrist in neutral position.

\section{DISCUSSION}

Dupuytren's disease is a benign fibroproliferative disease involving the palmar fascia, whereas carpal tunnel syndrome is a compressive neuropathy of the median nerve within the carpal tunnel underneath the transverse carpal ligament. Dupuytren contracture is mainly seen among the Celtic races with higher incidence among diabetics, epileptics and alcoholics. There may be a higher risk in patients with a history of trauma, smoking and HIV positive patients. In males, the age of onset is usually around the 5th decade but onset may manifest earlier among alcoholics and epileptics ${ }^{1-4}$. A review of the literature revealed no correlation between these two pathologies and we found no reported cases of these two conditions co-existing in the same patient. 


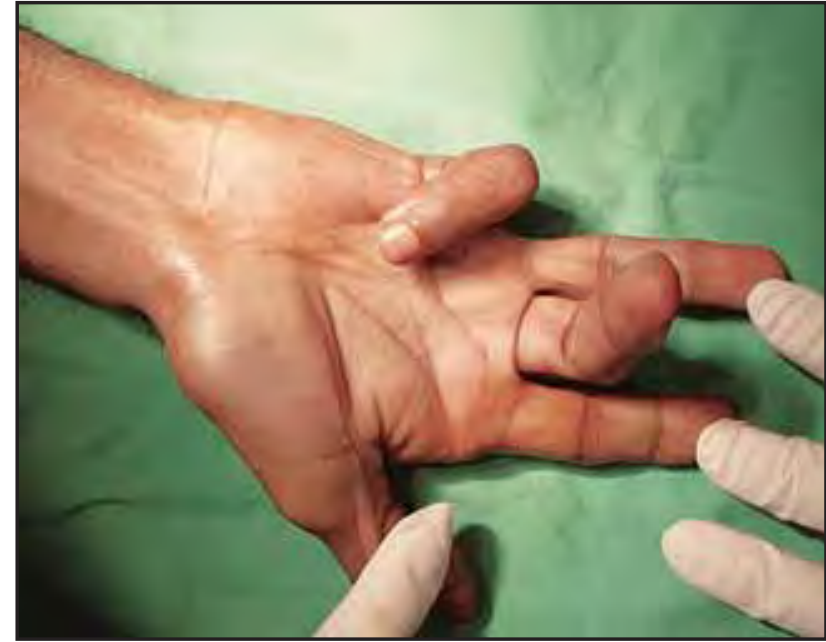

Fig. 1: Deformity over middle and little finger of the right hand.

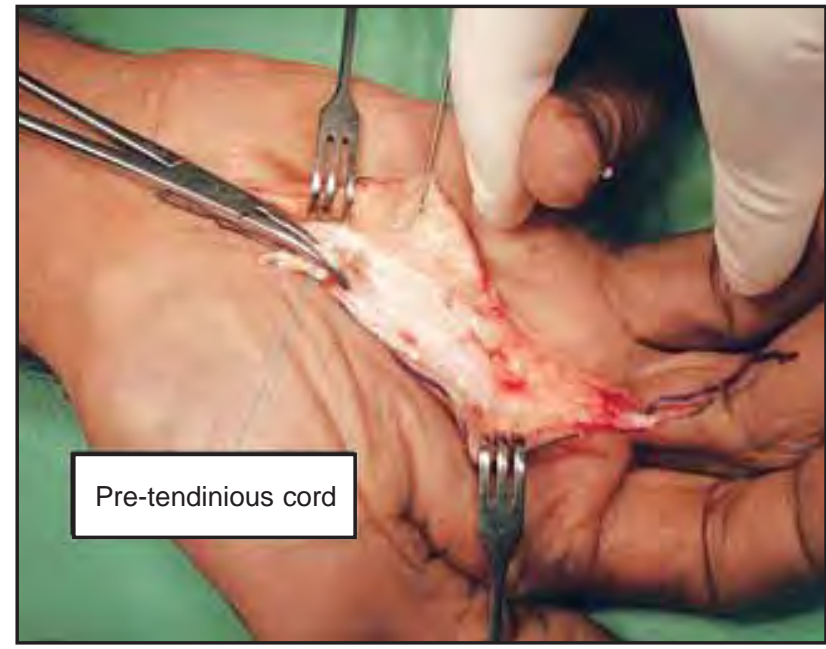

Fig. 3: Single zig zag palmar incision incorporated with carpal tunnel release incision.

Patients with Dupuytren's contracture involving digits can present with increasing numbness of the digit due to displacement of the neurovascular bundle by the spiral cord. The effect of the compressive symptoms may be more pronounced in the presence of proximal median nerve compression at the carpal tunnel. In this case, although the patient's main complaint was the increasing progressive flexion deformity of his finger, he also had numbness over the lateral three fingers but as this didn't interfere with his work, it was not brought to the attention of a doctor. Risk factors in this patient include chronic consumption of alcohol and multiple previous injuries to the right hand. The

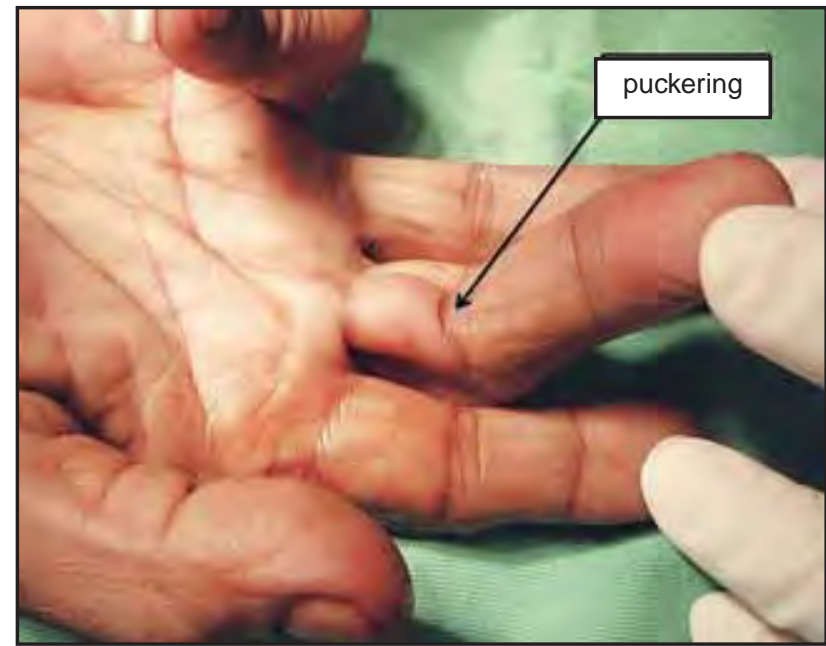

Fig. 2: Flexion deformity at PIP joint of right middle finger with puckering.

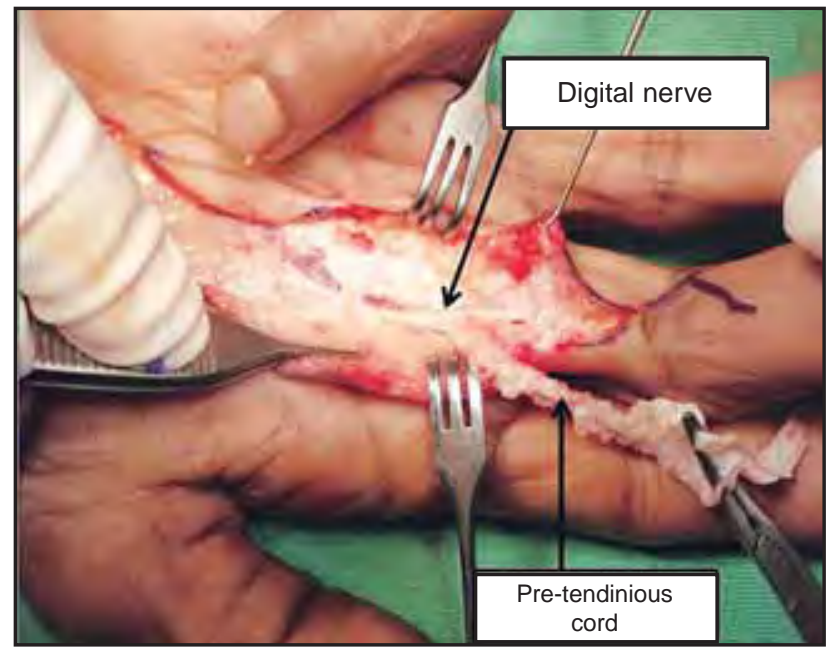

Fig. 4: Dissected pre-tendinious cord in relation to the digital nerve.

presence of little finger contraction over both hands can easily be mistaken for Dupuytren's disease but a thorough history and examination revealed that these deformities were present since birth and thus pointed to congenital camptodactyly; furthermore, on clinical examination there were no obvious cords felt or skin pitting seen. No treatment was offered for the camptodactyly as patient had no complaints or impaired function and it had been present since birth. 


\section{REFERENCES}

1. Smith RJ, Kaplan EB. Camptodactyly and Similar Atraumatic Flexion Deformities of the Proximal Interphalangeal Joints of the Fingers: A study of thirty-one cases. J Bone Joint Surg. 1968; 50A: 1187-249.

2. Ross DC. Epidemiology of Dupuytren's disease. Hand Clin. 1999; 15(1): 53-62.

3. Bradlow R, Mowat AG. Dupuytren's contracture and alcohol. Ann Rheum 1986; 45: 304-7.

4. Revis, DR Jr, Dupuytren Contracture. Available from URL: http://www.emedicine.com/med/topic592.htm Accessed 2008, September 23 . 